\title{
Pratiques de jachère et dispositifs d'appui en production bananière guadeloupéenne
}

\author{
Muriel BONIN*, Philippe CATTAN
}

Cirad, station de Neufchâteau, Sainte-Marie, 97130 Capesterre Belle Eau, FWI, France

muriel.bonin@cirad.fr

\section{* Correspondance et tirés à part}

Reçu le 11 janvier 2004 Accepté le 27 octobre 2005

Fruits, 2006, vol. 61, p. 83-98 (c) 2006 Cirad/EDP Sciences All rights reserved DOI: $10.1051 /$ fruits:2006007 www.edpsciences.org

RESUMEN ESPAÑoL, p. 98

\section{Practices of follow and support plans in banana production of Guadeloupe.}

Abstract - Introduction. In the Antilles, the introduction of fallow periods, whose utility in the control of telluric parasitism is proven, is on the agenda because of the parasitism explosion related to banana monoculture. As this practice is supported by research and the authorities, the purpose of the study undertaken was to analyze the differences between the recommendations set out by an institutional plan and farmers' practices in order to identify in which conditions fallow periods can be installed. Methods. The determinants and functions allotted to the fallow as well as the disincentives were approached starting with investigations concerning farmers. These made it possible to collect among other things the variables necessary for the characterization of the decision rules as regards crop allocation in farm land and endogenous exploitation constraints. The choice of the surveyed exploitations led us to study the practices of fallow within the framework of a typology distinguishing, among 42 exploitations studied, the types, "Diversified", "Banana exploitations family" and "Banana entrepreneurial". These types are representative of the exploitations of less than 20 ha. The case of the fallow period in Guadeloupe. The results showed that the fallow period is used by more than half of the surveyed farmers. Its situation varies with the exploitation type; it is either undergone, or integrated into the farming systems. The management systems are functions of the farmers' objectives: sanitation, pasture, cash management, etc. Disincentives of the fallow development are numerous: landlessness, difficulty in reasoning in the long term, loss of earnings on a land which "does not produce", etc. However, economic calculations on farming systems including fallows show that their gross margins are higher than in continuous and perennial banana plantations. The principal constraint is met at the time of the change of regime when the owner must face a land which will get an output supplement only later, in two years. Discussion and conclusion Our study highlighted that the multiplicity of the fallow practices, relevant for the owners' objectives, is only partly taken into account in the existing institutional devices. This calls for a diversification of the technical reference benchmarks.

France / Guadeloupe / Musa / fallow / cultivation / monoculture

\section{Pratiques de jachère et dispositifs d'appui en production bananière guadeloupéenne.}

Résumé - Introduction. Aux Antilles, l'introduction de la jachère, dont l'utilité dans la maîtrise du parasitisme tellurique est avérée, est à l'ordre du jour du fait de l'explosion du parasitisme lié à la monoculture bananière. Comme cette pratique est soutenue par la recherche et les pouvoirs publics, l'étude entreprise a eu pour but d'analyser les écarts entre les préconisations portées par un dispositif institutionnel et les pratiques des agriculteurs afin d'identifier à quelles conditions les pratiques de jachère peuvent être mises en place. Méthodologie. Les déterminants et fonctions attribués aux jachères ainsi que les freins ont été abordés à partir d'enquêtes auprès d'exploitants. Celles-ci ont permis de recueillir entre autres les variables nécessaires à la caractérisation des règles de décision en matière de gestion des assolements et de contraintes endogènes à l'exploitation. Le choix des exploitations enquêtées nous a conduits à étudier les pratiques de jachères dans le cadre d'une typologie d'exploitation distinguant, parmi 42 exploitations étudiées, les types, "Diversifié ", "Bananier familial " et "Bananier entrepreneurial ". Ces types sont représentatifs des exploitations de moins de 20 ha. Le cas de la jachère en Guadeloupe. Les résultats ont montré que la jachère est présente chez plus de la moitié des exploitants enquêtés. Sa situation varie avec les types d'exploitation ; elle est soit subie, soit intégrée dans les systèmes de culture. Les modes de conduites sont fonctions des objectifs des exploitants : assainissement, pâturage, gestion de la trésorerie, etc. Les freins au développement des jachères sont nombreux : manque de surface, difficulté de raisonner à long terme, manque à gagner sur une terre qui ne " produit " pas, etc. Cependant les calculs économiques sur des systèmes de culture incluant la jachère montrent que leurs marges brutes sont supérieures à celles trouvées pour des bananeraies continues et pérennes. La principale contrainte est rencontrée au moment du changement de système cultural lorsque l'exploitant doit faire face à une terre qui ne procurera un supplément de rendement que deux ans plus tard. Discussion et conclusion. Notre étude a mis en évidence que la multiplicité des pratiques de jachère, pertinentes au regard des objectifs des exploitants, n'est qu'en partie prise en compte dans les dispositifs institutionnels existants. Cela appelle une diversification des référentiels techniques.

France / Guadeloupe / Musa / jachère / pratique culturale / monoculture 


\section{Introduction}

La disparition des jachères et la mise en culture continue des sols est une des conséquences de l'accroissement de la productivité en agriculture. Pourtant, ce phénomène n'est pas irréversible et la jachère revient à l'ordre du jour, notamment en raison de l'émergence de questions environnementales et, en Europe occidentale, de contextes de surproduction. De fait, la question des conditions d'introduction de cette pratique dans les exploitations agricoles est posée. Elle fait référence au problème plus général de la relation entre l'évolution des pratiques agricoles et l'effet des politiques incitatives qui est parfois ambiguë. Dans le cas de la jachère, divers éléments font s'interroger sur la réussite de dispositifs de subvention sur l'installation de cette pratique :

- Les fonctions de la jachère sont multiples. Une définition simple de la jachère est proposée par Sebillotte [1] : " La jachère est l'état de la terre d'une parcelle entre la récolte d'une culture et le moment de la mise en place de la culture suivante. "Les problématiques liées à la jachère se complexifient compte tenu de la grande diversité des fonctions de la jachère et de la variété des modalités de conduite selon les exploitations et les milieux écologiques [2]. Restaurer la fertilité, alimenter un troupeau pour une jachère enherbée et pâturée, moyen de retrait de terres cultivées pour limiter la production en céréales, oléagineux et protéagineux avec la réforme de la Politique agricole commune en 1992 : autant d'objectifs différenciés, dans des contextes variés, qui induisent des problématiques diverses et qui ne permettent pas d'envisager un développement des surfaces en jachère sans s'intéresser à celui de ses fonctions associées.

- Ces fonctions sont en parties déterminées par des facteurs liés à la pression foncière. En zones tropicale et méditerranéenne, les modalités de pratique de la jachère sont très diverses. Pour Jouve [3], l'examen de cette diversité confirme l'hypothèse de Boserup [4] qui considère la jachère comme un indicateur particulièrement discriminant des modes d'exploitation du milieu et de leur évolution en fonction de l'accroissement de la densité de population et du taux d'occupation du sol. La disponibilité foncière est en effet une des conditions indispensables à la mise en jachère qui est compromise lorsque la pression démographique s'accroît.

- Il existe très souvent un écart entre les objectifs des dispositifs d'appui ${ }^{1}$ et ceux des agriculteurs comme le montrent Bellon et al. [5] à partir d'enquêtes sur les pratiques de jachère dans des contextes de France métropolitaine contrastés (Préalpes, Brie laitière, Vexin). Le gel de terres arables conditionnant l'accès aux paiements compensatoires européens s'inscrivait dans des objectifs de limitation de la surproduction. Les pratiques mises en ouvre par les agriculteurs (par exemple jachère ayant pour fonction de faciliter l'organisation du travail, donner de la souplesse dans le système d'alimentation pour les éleveurs, permettre un labour précoce pour les cultivateurs) ont des objectifs éloignés de ceux à l'origine des mesures accompagnant le gel des terres.

Ces expériences montrent la complexité des relations entre les décisions extérieures à l'exploitation et les pratiques agricoles et posent la question de la réduction des écarts entre préconisations et pratiques des agriculteurs.

Cette question est d'actualité aux Antilles françaises, où l'explosion du parasitisme (insectes, nématodes, etc.) lié à la monoculture de la banane sur une longue période [6] et les atteintes à l'environnement découlant de la lutte chimique appliquée sur la culture posent également la question de l'introduction de la jachère. Son utilité dans la mâtrise du parasitisme tellurique est avérée en culture bananière [7-9], comme pour d'autres cultures [10] et a conduit à promouvoir son développement. Celui-ci s'est appuyé, dans un premier temps, sur la définition d'un message technique adossé à des subventions : des fiches techniques ont été élaborées $[11,12]$ et des aides ont été introduites en s'appuyant sur différents dispositifs

\footnotetext{
${ }^{1}$ Nous retenons ici une définition restrictive de la notion de " dispositif d'appui " qui est utilisée dans cet article pour regrouper les référentiels techniques et les subventions destinées à encourager les pratiques de jachère.
} 
institutionnels $\left(\mathrm{CTE}^{2}, \mathrm{CAD}^{3}, \mathrm{DOCUP}^{4}\right.$, etc.). Or les exemples précédents font s'interroger sur la réussite d'un tel dispositif.

Notre article se propose d'une part d'analyser la nature des écarts existant entre les préconisations portées par un dispositif institutionnel et les pratiques des agriculteurs, d'autre part d'identifier les améliorations à apporter dans la construction des dispositifs d'appui. Le cas de la pratique des jachères en Guadeloupe a été retenu. Après avoir dressé le contexte d'élaboration du message technique porté par les institutionnels et la façon dont il prend en compte les attentes de chacun, nous nous sommes interrogés plus particulièrement sur les déterminants et les fonctions attribués aux jachères par les agriculteurs, puis sur les freins aux pratiques de jachère (agronomique ou technico-économique) limitant leur mise en place. La prise en compte de ces résultats dans la construction de dispositifs d'appui sera ensuite discutée.

\section{Méthodologie}

\subsection{Cadre méthodologique}

Les déterminants et fonctions attribués aux jachères ainsi que les freins ont été abordés à partir d'enquêtes auprès d'exploitants. La jachère peut être considérée comme un cas particulier de rotation. D'autre part, la rotation oblige parfois à une période de jachère en raison d'incompatibilités entre cultures. L'étude des freins à l'adoption de jachère conduit donc également à s'intéresser à la rotation.

Les variables nécessaires à la caractérisation des déterminants de la mise en place de la jachère sont issues d'une formalisation du processus de gestion pluriannuelle des

\footnotetext{
${ }^{2}$ Les Contrats Territoriaux d'Exploitation (CTE) sont un outil de la loi d'orientation agricole française de 1999 ; ils visent à prendre en compte les fonctions économiques, environnementales et sociales de l'agriculture.

${ }^{3}$ Les CAD (Contrats d'Agriculture Durable) remplacent les CTE depuis 2003.

${ }^{4}$ DOCUP =DOCument Unique de Programmation.
}

assolements par l'exploitant, proposée par Papy [13]. Plusieurs groupes de variables sont distingués. Un premier ensemble porte sur l'identification des règles de décision conduisant à l'implantation d'une culture sur une parcelle donnée. On distingue :

- (1) des règles par culture ayant trait à la localisation (où peut aller la culture ?), au dimensionnement (quelle surface peut occuper cette culture ?) à la pertinence technique des successions culturales (quels sont les précédents possibles? quel est le nombre maximum de retours successifs de la culture sur elle même et les délais de retour ?) ;

- (2) des règles d'arbitrages entre cultures ayant trait au choix d'orientation de production, aux priorités d'attribution des moyens de production aux cultures.

Ces règles sont soumises à un certain nombre de contraintes qui constituent le second ensemble de variables. Deux types de contraintes sont distingués :

- (1) des contraintes endogènes au fonctionnement de l'exploitation (ressources en terre, eau, équipement, main d'œuvre, productions et activités, etc.) ;

- (2) des contraintes exogènes de nature économique (organisation des marchés, débouchés, etc.), législative ou contractuelle (législation sur l'usage des produits phytosanitaires autour des captages d'eau, mesures et actions de soutien technique et financier, usages non agricoles de l'espace, etc.).

Une évaluation économique a été entreprise à partir des caractéristiques chiffrées recueillies au cours des enquêtes. Les référentiels provisoires (données économiques) acquis en zone bananière dans le cadre de la mise en place du modèle MICA [14] ont été utilisés. Ils ont été construits sur la base d'enquêtes directes qui ont permis d'évaluer produits et charges de différents modes de conduite du bananier ${ }^{5}$, notamment à partir

\footnotetext{
${ }^{5}$ Sont pris en compte les coûts suivants par hectare et par an : destruction de la bananeraie, plantation, fertilisation, désherbage, traitements phytosanitaires, façons culturales, soins aux régimes, irrigation, récolte, conditionnement et expédition, entretien et aménagement fonciers, amortissements et frais financiers.
} 
d'une évaluation des coûts des différents actes techniques. Les amortissements et frais financiers ont été retranchés de la marge brute ; par commodité, ce résultat a été nommé "marge brute" dans la suite du texte. Ces référentiels ont été ajustés aux données d'enquêtes pour plusieurs paramètres ${ }^{6}$ spécifiques aux exploitations étudiées.

\subsection{Guide d'entretien}

Les enquêtes ont permis de recueillir les variables utiles à notre propos, notamment celles nécessaires à la caractérisation des règles de décision en matière de gestion des assolements et aux contraintes endogènes à l'exploitation. Les enquêtes ont été de type semi-directif et axées sur quatre thèmes :

- (1) le système de production et la stratégie globale de l'exploitant qui a pour objectif de rattacher les agriculteurs à la typologie préexistante, d'identifier les objectifs de l'agriculteur ainsi que les raisons de ses choix ; les fonctions attribuées à la jachère y sont notamment relevées ;

- (2) les différents systèmes de culture qui permettent d'expliciter les itinéraires techniques des différentes cultures et les règles de succession entre culture ;

- (3) la coordination des systèmes qui permet d'identifier au sein de l'exploitation les règles d'attribution des ressources productives (terre, main d'œuvre, trésorerie) aux cultures ;

- (4) les rotations culturales. Il s'agit ici de caractériser une série de contraintes endogènes à partir du point de vue du producteur sur les rotations culturales, leur intérêt et désavantage à la lumière de sa pratique ou d'informations diverses, les freins à leur adoption.

\section{3. Échantillonnage des exploitations}

La nécessité d'aboutir à un compromis entre l'exhaustivité des enquêtes et les moyens

${ }^{6}$ Coûts de destruction de la bananeraie, de la plantation, de la fertilisation, des traitements phytosanitaires; tonnage expédié (lié au rendement). Ces paramètres varient selon les systèmes de culture (tableau III). disponibles a conduit à sélectionner un nombre limité de territoires et d'exploitants avec l'objectif principal de capter la diversité des déterminants, freins et fonctions de la jachère.

Du point de vue territorial, les enquêtes ont été limitées à la commune de Capesterre-Belle-Eau, située en côte au vent de la Basse-Terre et qui possédait, en 2000, à elle seule, $54 \%$ des surfaces en bananeraies de la Guadeloupe [15]. Les enquêtes ont été effectuées dans trois petites régions agricoles de la commune, choisies pour la diversité de leur territoire, préalablement mise en évidence par des diagnostics territoriaux réalisés en 2000 et 2001 [16-18]. Il s'agit de la rive nord du bassin-versant de la rivière Pérou caractérisée par la dominance de la culture du bananier et la présence d'andosols ; des sections de La Sarde (SainteMarie), soumise à une forte pression foncière du fait de l'urbanisation croissante et caractérisée par des cultures sur sol ferrallitique et des problèmes de sécheresse récurrents ; de l'Habituée, zone de passage à destination d'un des principaux sites touristiques de l'île, caractérisée par des sols possédant une forte pierrosité.

Le choix des exploitations enquêtées s'est appuyé sur la démarche proposée par Landais [19] et nous a conduits à étudier les pratiques de jachères dans le cadre d'une typologie d'exploitation précédemment établie [20] distinguant :

- le type "Polyculture-élevage " de très faibles surfaces [(1 à 5) ha], produisant essentiellement des cultures vivrières et fruitières destinées à l'autoconsommation et au marché local ;

- le type "Diversifié " produisant de la "banane export " et d'autres cultures destinées au marché local (vivrier, maraîchage, fruits, fleur) ;

- le type "Bananier familial ", de surface réduite [(5 à 10) ha] et ne produisant pratiquement que de la "banane export ";

- le type "Bananier entrepreneurial ", bien pourvu en facteurs de production ;

- le type "Sociétés bananières" dont le capital n'est pas entièrement détenu par l'exploitant. 
Tous les types n'ont pas été étudiés. L'étude a été restreinte aux exploitations produisant de la banane pour l'exportation ; le dispositif a exclu les types extrêmes "Polyculture-élevage ", peu orienté vers la monoculture, et "Société bananière ", en raison de la complexité des centres de décision - pour retenir 42 exploitations parmi les types, "Diversifié ", "Bananier familial " et "Bananier entrepreneurial ". Ces types sont représentatifs des exploitations de moins de 20 ha, qui représentaient, en 1997, $93 \%$ des exploitants, $66 \%$ des surfaces et $58 \%$ des tonnages [21], favorisant de fait la représentativité des structures d'exploitation plutôt que leur poids économique.

Le choix des exploitations à enquêter s'est fait aléatoirement à l'intérieur de chaque zone, parmi les types retenus. L'objectif principal portant sur la caractérisation des types et de la diversité des comportements, l'échantillon final est apparu relativement équilibré entre les trois types retenus et n'a donc pas été représentatif de l'importance des types au sein des exploitations bananières de Guadeloupe. Finalement, l'échantillon a été composé pour $21 \%$ du type "Bananier entrepreneurial ", $45 \%$ du type "Bananier familial " et $34 \%$ du type "Diversifié ".

\section{Le cas de la jachère en Guadeloupe}

\section{1. Élaboration du message technique}

Les systèmes de culture majoritairement pratiqués en zone de production bananière guadeloupéenne sont basés sur la culture du bananier en continu, entrecoupée de périodes d'interruption avant replantation, volontaires (baisse de rendement entraînant la décision de replantation) ou non (cyclones, sécheresse, parasitisme entraînant la destruction des plantations). Or, la monoculture fragilise la bananeraie - en favorisant notamment la prolifération de parasites telluriques et aériens et en diminuant la fertilité physico-chimique des sols [22] - et tend à augmenter la fréquence de replantation. Ces constats ont conduit à mettre au point des techniques d'assainissement et de restauration de la fertilité au cours des années quatre-vingt. Ainsi, différents travaux ont montré l'intérêt d'introduire la jachère ou d'autres cultures en rotation [2325], notamment pour maîtriser le parasitisme de façon à réduire l'utilisation des pesticides.

La mise en évidence de pollutions de captages d'eau en 2000 conduisant parfois à leur fermeture et la médiatisation de ces événements [26] ont catalysé l'émergence de la problématique environnementale dans l'opinion publique en Guadeloupe. L'extension des contaminations à des cultures traditionnelles telles que la patate douce (Ipomoea batatas), le madère (Colocasia esculenta) ou encore l'igname (Dioscorea spp.) a suscité l'inquiétude de l'opinion publique [27]. La production bananière a été accusée au premier plan et plusieurs rapports ministériels ont souligné les risques de pollution par les pesticides, notamment associés à la culture du bananier [28-30]. La diminution de la charge phytosanitaire est devenue une urgence. Reprenant les conclusions des travaux précédents, ce nouvel objectif a conduit à la mise au point d'une fiche technique " jachère raisonnée " basée sur le maintien d'une couverture morte durant une période minimale d'un an - en utilisant un herbicide, le glyphosate - pour éviter le développement des nématodes sur d'éventuelles plantes hôtes. L'efficacité de la destruction des bananiers par injection de glyphosate [31] pour la réduction des populations de nématodes a justifié la vulgarisation de cette technique. Parallèlement, un dispositif d'alerte au traitement nématicide basé sur le suivi des populations a été mis en place. Il permet de différer de près d'un an le premier traitement nématicide après plantation de la bananeraie succédant à la jachère.

Ainsi, la prise en compte du risque environnemental lié à l'application de produits phytosanitaires, et plus généralement au maintien de la fertilité des parcelles, s'est traduite par une évolution des fonctions dévolues à la jachère. L'efficacité dans la lutte contre le parasitisme apparaît renforcée ; des fonctions nouvelles sont associées à la pratique (restructuration des sols, correction des carences), alors que d'autres disparaissent (fonction de pâturage entre autres en 
Tableau I.

Taux d'occupation du sol du bananier en fonction de la surface agricole utile et selon trois types d'exploitations [20] visitées en Guadeloupe.

$\begin{array}{lccccc}\begin{array}{l}\text { Type } \\ \text { d'exploitation }\end{array} & \begin{array}{c}\text { Nombre } \\ \text { d'exploitations }\end{array} & \begin{array}{c}\text { Surface agricole utile (SAU) } \\ \text { moyenne par exploitation } \\ \text { (ha) }\end{array} & \begin{array}{c}\text { \% moyen des surfaces } \\ \text { en monoculture } \\ \text { par rapport à la SAU }\end{array} & \begin{array}{c}\text { moyen des surfaces } \\ \text { en bananiers }\end{array} & \begin{array}{c}\% \text { moyen des surfaces } \\ \text { par rapport à la SAU diversification }\end{array} \\ \text { par rapport à la SAU }\end{array}$

\section{Tableau II.}

Nombre d'exploitations agricoles pratiquant la jachère, volontaire ou accidentelle, selon trois types d'exploitation étudiés en Guadeloupe.

\begin{tabular}{|c|c|c|c|c|c|}
\hline $\begin{array}{l}\text { Type } \\
\text { d'exploitation }\end{array}$ & $\begin{array}{l}\text { Nombre } \\
\text { d'exploitations } \\
\text { agricoles }\end{array}$ & $\begin{array}{c}\text { Nombre } \\
\text { d'exploitations } \\
\text { agricoles pratiquant } \\
\text { la jachère }\end{array}$ & $\begin{array}{l}\text { \% moyen des surfaces en } \\
\text { jachère par rapport à la surface } \\
\text { agricole utile dans les } \\
\text { exploitations la pratiquant }\end{array}$ & $\begin{array}{l}\text { Nombre d'exploitations } \\
\text { agricoles pratiquant la } \\
\text { jachère volontaire }^{1}\end{array}$ & $\begin{array}{c}\text { Nombre d'exploitations } \\
\text { agricoles pratiquant la } \\
\text { jachère } \\
\text { accidentelle }\end{array}$ \\
\hline $\begin{array}{l}\text { Bananier } \\
\text { entrepreneurial }\end{array}$ & 9 & 6 & 22 & 6 & 2 \\
\hline $\begin{array}{l}\text { Bananier } \\
\text { familial }\end{array}$ & 19 & 10 & 26 & 4 & 9 \\
\hline $\begin{array}{l}\text { Culture } \\
\text { diversifiée }\end{array}$ & 14 & 8 & 26 & 4 & 4 \\
\hline
\end{tabular}

raison de l'instauration d'une couverture morte). On passe du concept de période intermédiaire entre deux cultures à une pratique de haute technicité introduisant un ensemble de règles notamment sur la gestion du couvert végétal (en particulier enlèvement des repousses de bananier), sur le type d'interventions en parcelle et sur la durée de la période.

Des référentiels techniques introduisant une certaine technicité dans la conduite de la jachère ont donc été élaborés. Qu'en estil dans les exploitations agricoles?

\subsection{La jachère et ses déterminants dans les exploitations agricoles}

Les exploitations agricoles qui ont été étudiées sont de surface modeste (tableau I) comme la plupart des propriétés de la zone bananière guadeloupéenne [21]. La mono- culture (bananière et autres cultures) est dominante dans ces plantations : elle occupe en moyenne $63 \%$ de la surface agricole utile des exploitations visitées. Même dans les types diversifiés, la présence de plusieurs cultures sur une propriété n'implique pas leur succession sur une même parcelle et la coexistence de plusieurs systèmes monoculturaux est fréquemment observée au sein des exploitations. Les surfaces en banane (monoculture et rotation) occupent en moyenne $70 \%$ des surfaces des exploitations soumises à nos enquêtes. Au moment de nos investigations, la jachère n'était présente que sur 24 des 42 exploitations étudiées (tableau II). Globalement, elle apparaît un peu plus fréquemment chez les "Bananiers entrepreneuriaux" (chez $67 \%$ de ce type d'exploitant), contre (53 et 57) \% chez les deux autres types considérés. En revanche, en ne considérant que les jachères volontaires, les pourcentages d'exploitation 
Tableau III.

Nombre d'exploitations agricoles pratiquant la jachère pour des raisons d'ordre agronomique ou économique en fonction de trois types d'exploitation du bananier, étudiés en Guadeloupe.

\begin{tabular}{|c|c|c|c|c|c|c|}
\hline \multirow{2}{*}{$\begin{array}{l}\text { Type } \\
\text { d'exploitation }\end{array}$} & \multirow{2}{*}{$\begin{array}{l}\text { Nombre d'exploitations agricoles } \\
\text { pratiquant la jachère }\end{array}$} & \multicolumn{3}{|c|}{ Raisons agronomiques } & \multicolumn{2}{|c|}{ Raisons économiques } \\
\hline & & $\begin{array}{l}\text { Assainissement } \\
\text { (vitroplants) }\end{array}$ & Pâturage & Fertilité & $\begin{array}{l}\text { Adaptation } \\
\text { production }\end{array}$ & $\begin{array}{l}\text { Replantation } \\
\text { échelonnée }\end{array}$ \\
\hline $\begin{array}{l}\text { Bananier } \\
\text { entrepreneurial }\end{array}$ & 6 & 5 & 1 & - & - & - \\
\hline Bananier familial & 10 & 2 & - & - & 6 & 2 \\
\hline Diversifié & 8 & 3 & - & 1 & 2 & 2 \\
\hline
\end{tabular}

ayant ce type de jachère chez les " Bananiers familiaux " et les "Diversifiés " tombent respectivement à (21 et 29) \%, alors qu'ils restent à $67 \%$ pour le type "Entrepreneurial ". Quand la jachère est présente, elle occupe en moyenne plus de $20 \%$ de la surface agricole utile avec des variations atténuées entre types d'exploitations (tableau II).

Les raisons évoquées à l'origine de la mise en place de la jachère (tableau III) peuvent être d'ordre économique. Il s'agit alors de la diminution de la surface cultivée, soit volontaire par exemple pour adapter le volume de production à la main-d'œuvre disponible, soit subie du fait d'accidents climatiques associés à une replantation échelonnée résultant d'un manque de maind'œuvre ou de trésorerie. Les raisons de type économique priment chez les types diversifiés et surtout familiaux, les plus affectés par la réduction de surface (tableau III). Si la décision d'implanter une nouvelle culture est d'ordre économique (obtention d'un complément de revenu), la décision d'instaurer des rotations relève en premier lieu d'un choix agronomique (assainissement du sol, amélioration de la fertilité) pouvant se traduire par un gain économique. De même, la mise en place volontariste des jachères au sein des systèmes de culture résulte d'une décision agronomique. Ces décisions sont le plus souvent supportées par un conseil technique, notamment en relation avec l'utilisation de vitroplants de bananiers pour lesquels l'assainissement préalable du sol est nécessaire. Il peut encore s'agir de pâturage. D'autre part, la diversification adoptée par certains agriculteurs les oblige également à passer parfois par une période de jachère en raison d'incompatibilité entre cultures. Par exemple, deux exploitants que nous avons rencontrés pratiquent les rotations suivantes : "banane/ jachère/igname " et "banane/jachère/marâ̂chage/jachère ". L'introduction d'une jachère entre banane et igname est alors motivée par des risques parasitaires : les nématodes du bananier pourraient avoir des effets sur l'igname. Cependant, notre enquête a montré que les producteurs manquaient de références sur les règles de successions culturales (précédents possibles, délais de retour d'une culture sur elle-même, etc.).

En conclusion, on constate que la jachère est présente chez plus de la moitié des exploitants où elle occupe des surfaces importantes. La situation de la jachère varie profondément avec les types d'exploitation. Chez les "Bananiers familiaux " où la monoculture domine, la jachère est subie et reflète les difficultés de l'exploitation agricole. Les deux autres types d'exploitation considérés par notre étude sont moins exposés aux aléas économiques de la filière: le type "Entrepreneurial " du fait de sa surface plus importante, et le type "Diversifié " du fait des ses orientations culturales. Ces deux derniers types d'exploitation intègrent la jachère dans leur système de culture notamment en raison de la reconnaissance de ses effets agronomiques.

\subsection{Diversité des modes de conduite de la jachère}

Les éléments évoqués précédemment déterminent différents modes de conduites de la 


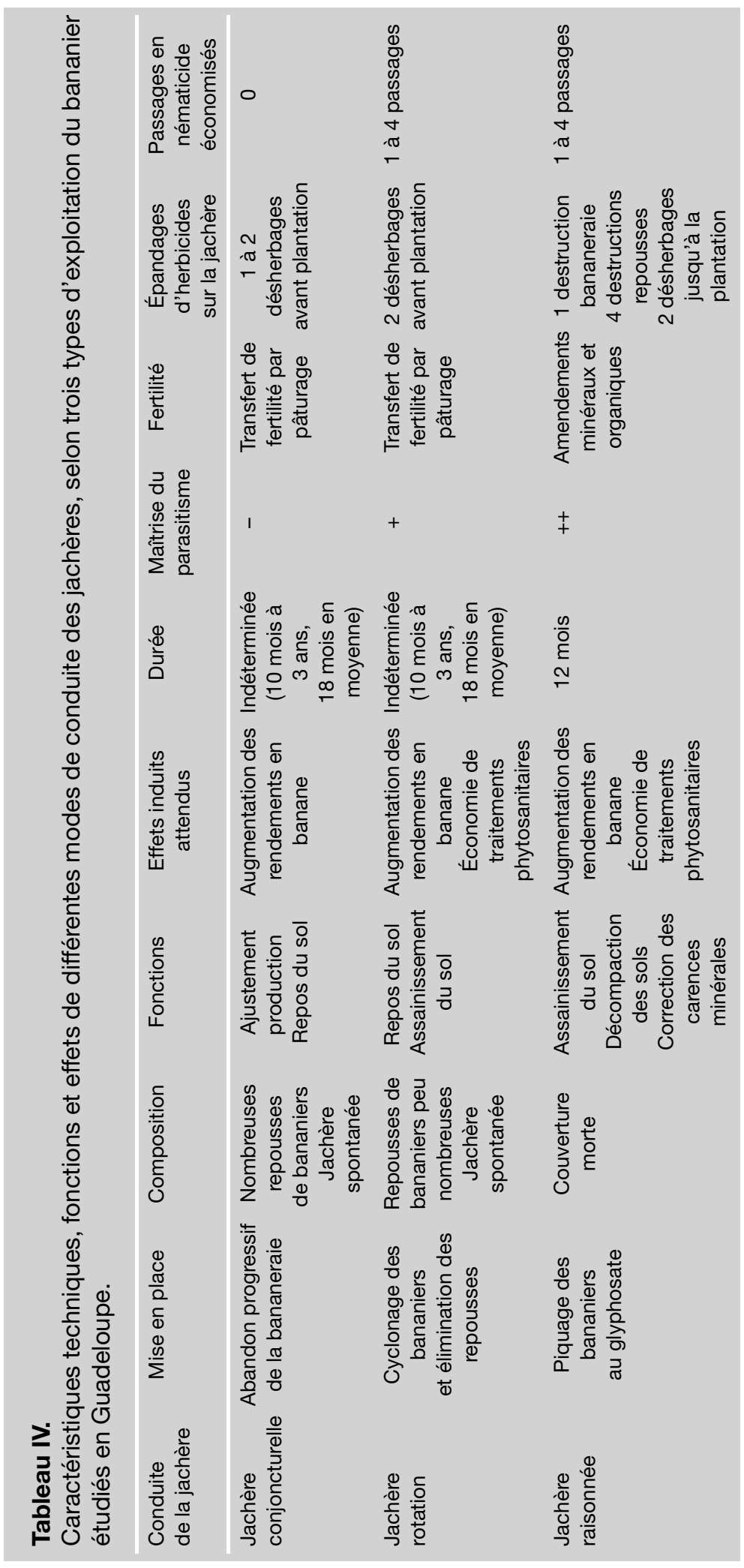

jachère qui correspondent à différents degrés d'intégration de la jachère dans le système de culture, à différents niveaux techniques, ainsi qu'à différentes fonctions et attentes. Parmi les types de jachère observés dans les systèmes de culture incluant le bananier (tableau IV), les jachères de type " Raisonnée " et " Rotation " sont volontaires. Le passage de la jachère "Conjoncturelle " à la jachère "Raisonnée " se traduit par une augmentation de la technicité liée à la conduite de la jachère, alors que les fonctions qui lui sont attribuées s'élargissent (tableau IV). La durée de la jachère ne constitue pas un facteur limitant de son adoption, alors que la pratique l'établit en moyenne à 18 mois. Par ailleurs, jachère n'est pas synonyme d'efficacité dans la maîtrise du parasitisme, notamment pour la jachère "Conjoncturelle " où cette fonction n'est pas considérée. Enfin, il apparaît que la prise en compte d'une fonction d'assainissement, destinée à limiter l'emploi de nématicides, se traduit par une utilisation accrue d'herbicide (tableau IV). Un gain environnemental est toutefois attendu du fait de l'utilisation de produits moins nocifs : par exemple la "Dose Journalière Admissible " est de 1000 fois plus élevée pour le glyphosate que pour un nématicide comme le cadusafos. Finalement, la notion de régénération de la fertilité est une composante forte de la jachère chez les agriculteurs qui en connaissent l'intérêt; elle justifie le pâturage pour certains exploitants, pratique non compatible avec le maintien d'une couverture morte sur la jachère "raisonnée".

La variabilité des déterminants de la mise en place d'une jachère conduit à une grande diversité des pratiques. Il reste qu'une majorité des surfaces est en monoculture. Quels sont alors les freins au développement des interruptions culturales? Cette question fait appel à des règles par culture et des arbitrages entre cultures [13].

\subsection{Freins à l'adoption de jachères et rotations}

Nos enquêtes effectuées dans les exploitations guadeloupéennes ont montré que le manque de connaissance sur les effets agronomiques des jachères et rotations n'était 
pas un frein majeur : seulement $22 \%$ des producteurs interrogés n'en connaissent pas l'intérêt ou pensent qu'elles sont inutiles ; $45 \%$ d'entre eux sont convaincus de leur intérêt, mais ne les pratiquent pas ; $33 \%$ sont convaincus de leur utilité et les pratiquent. En revanche, l'effet du manque de surfaces agricoles utiles et de surfaces agricoles utiles mécanisables [25] a été confirmé. Ces observations confirment l'hypothèse, posée en introduction, de la nécessité de disponibilité foncière comme condition à la mise en jachère. Ainsi, il n'a pas été observé de pratique de jachère ou de rotation dans des exploitations de moins de 5 ha. Ce seuil passe à 10 ha pour des exploitations ayant moins de $50 \%$ de surfaces agricoles utiles mécanisables (figure 1). Nous constatons également que la présence de rotations est liée à la possibilité de mécanisation (absence de rotation lorsque moins de $50 \%$ de la surface agricole utile est mécanisable).

Les freins à l'adoption de pratiques de jachère et de rotation peuvent être en rapport avec la stratégie productive de l'exploitant. Les agriculteurs en phase de diversification n'ont pas d'objectif à long terme sur la culture du bananier et ne privilégient donc pas les pratiques de jachère ou de rotation. Pour les planteurs dont la production de banane reste l'objectif principal, les freins sont de divers ordres : manque à gagner sur une terre non cultivée ; frein psychologique de voir une terre non travaillée ; manque de références techniques. D'autres agriculteurs enfin associent, dans leur système de production, le bananier et des cultures de diversification, susceptibles de constituer un apport en trésorerie à certains moments clés pour la culture du bananier ou dans la trajectoire des exploitations. Ces combinaisons de productions ne se traduisent toutefois pas toujours par une mise en rotation et on observe ainsi des juxtapositions de monocultures au sein d'une même exploitation. Les freins à la combinaison de cultures dans une rotation peuvent ici porter sur un manque de connaissance de leurs effets agronomiques. D'autre part, la diversification peut ne répondre qu'à des objectifs économiques de complément de revenu, l'assainissement des parcelles vis-à-vis des parasites n'étant pas un objectif prioritaire du producteur.

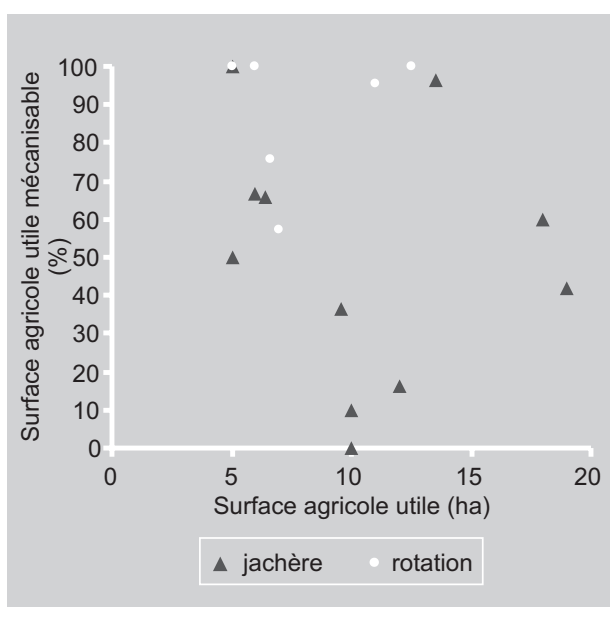

Figure 1.

Répartition des exploitations cultivant le bananier en alternance avec la jachère ou d'autres cultures (rotation), selon leur superficie agricole utile et le taux de superficie agricole utile mécanisable (Guadeloupe).
Les freins à la mise en place de rotations peuvent également porter sur des facteurs ayant trait à la gestion des assolements à l'échelle de l'exploitation et notamment sur des contraintes spécifiques de localisation des cultures empêchant un changement d'affectation des parcelles. Ainsi, les cultures de diversification font souvent l'objet de règles de localisation précises : madère en zone humide, igname à proximité de l'habitation pour éviter le vol, ananas dans les zones accidentées et difficilement accessibles (la production étant plus facile à transporter que les régimes de banane). La localisation de la jachère pâturée est raisonnée non seulement en fonction de critères agronomiques, mais aussi et surtout en fonction des risques de vol des animaux qui la pâturent (proximité d'une voie de circulation, d'un point de surveillance, etc.). En revanche, la bananeraie est rarement localisée de manière spécifique. Pour $67 \%$ des exploitants interrogés, la culture du bananier peut " aller partout " dans leur parcellaire ; les $33 \%$ restants localisent leur bananeraie sur les terres les plus accessibles et les moins accidentées afin de limiter le transport des régimes et les chocs lors de ces transports. L'importance de cette contrainte de localisation pour les exploitants interrogés a été évaluée à partir de l'identification de trois comportements type : "localisation indifférente "; "localisation d'une culture particulière " : une culture fait l'objet d'une règle de localisation stricte, la localisation des autres est indifférente ; "localisation spécifique ": chaque culture de l'exploitation a 


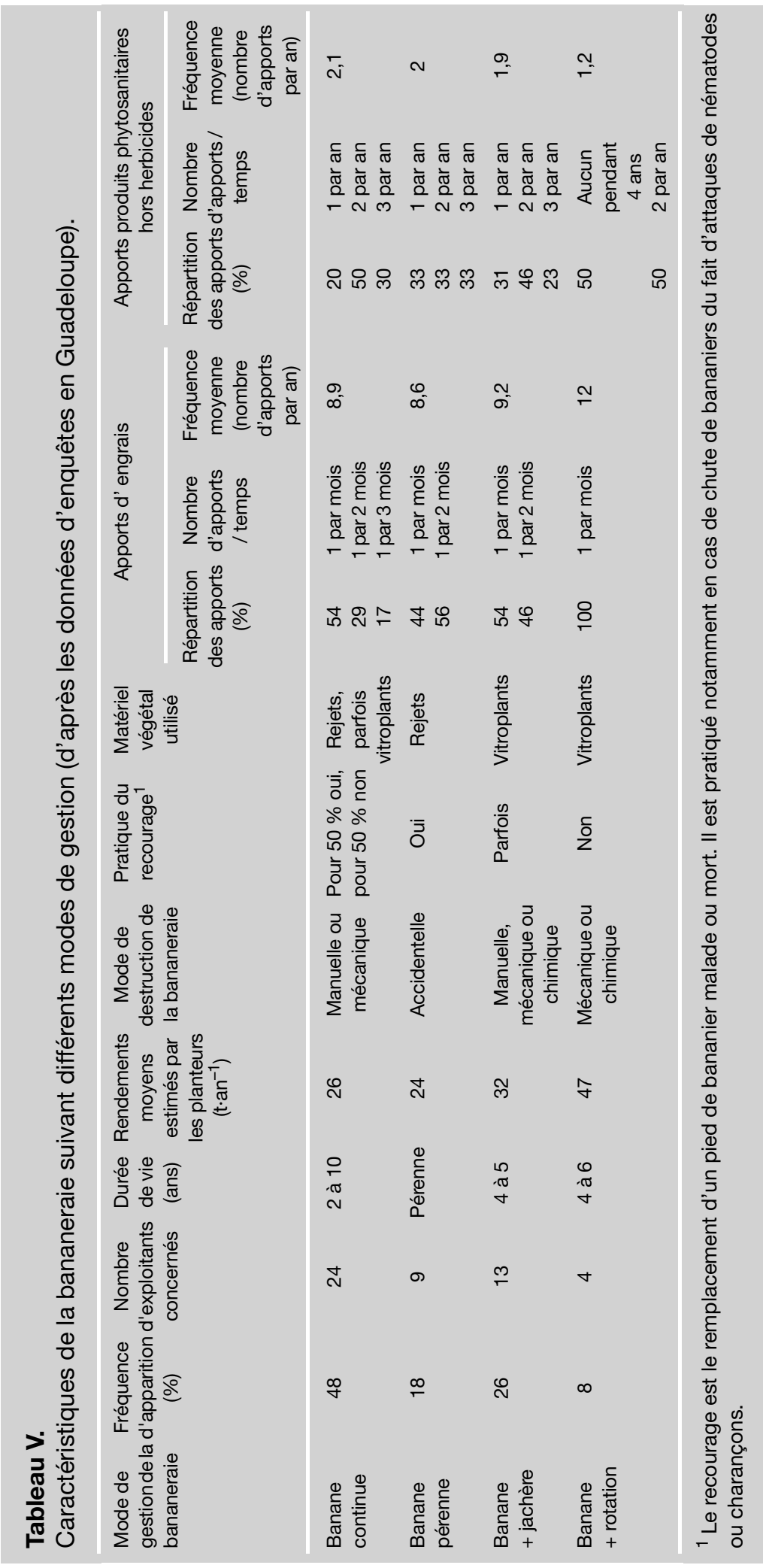

une règle de localisation précise. La répartition dans l'échantillon d'enquête a été respectivement de $(52,19$ et 29$) \%$; la localisation spécifique est donc une des causes de l'absence de rotation dans une exploitation diversifiée, mais ce n'est pas la seule.

Pour conclure, nous constatons que le niveau de la parcelle ne suffit pas pour appréhender les freins aux pratiques de jachères et rotations. Si la pertinence du niveau " exploitation " est avérée (objectifs économiques de l'exploitant, stratégie, etc.), il ne suffit pas non plus. En effet, jachère et rotation impliquent des questions de gestion de l'espace par les agriculteurs [5]. Or, en Guadeloupe, zone insulaire où l'espace est limité et où de multiples usagers doivent cohabiter sur un espace restreint, les interactions entre acteurs pour la gestion de cet espace conditionnent particulièrement les activités agricoles. Ainsi le manque de surfaces agricoles utiles, lié en partie à la pression foncière, détermine la mise en place de systèmes de culture incluant la jachère et la rotation. D'autre part, les règles de localisation spécifique des cultures font souvent intervenir d'autres usagers de l'espace : par exemple les risques de vols qui sont fréquents en milieu périurbain. La prise en compte des interactions entre agriculteurs et autres usagers de l'espace est en conséquence nécessaire, notamment en caractérisant les alliances et conflits pour l'usage de cet espace [32].

Il existe donc un ensemble de facteurs justifiant ou freinant l'adoption de jachères. L'impact sur le revenu et la marge brute dégagée par les cultures est également un de ces facteurs qu'il est nécessaire d'aborder.

\section{5. Évaluation économique}

Les modes de gestion de la bananeraie diffèrent selon les systèmes de culture. Aux dires des exploitants, le rendement de la banane augmente nettement dans des systèmes avec jachère ou rotation, comparativement à la bananeraie continue ou à la bananeraie pérenne (tableau V). De plus, les apports de produits phytosanitaires, hors herbicides, sont moins fréquents.

Les marges brutes annuelles par hectare, calculées pour les cultures de bananier 
Tableau VI.

Gain économique (différence de marge brute) en $€ \cdot h \mathrm{a}^{-1} \cdot \mathrm{an}^{-1} \mathrm{du}$ mode de conduite « banane + jachère » par rapport aux systèmes " banane continue » et « banane pérenne » en fonction du niveau de subventions (Guadeloupe).

\begin{tabular}{|c|c|c|c|}
\hline \multirow[t]{2}{*}{ Conduite " Banane + jachère » } & \multirow[t]{2}{*}{$\begin{array}{c}\text { Gain par rapport } \\
\text { à la « banane continue » } \\
\left(€ \cdot \mathrm{ha}^{-1} \cdot \mathrm{an}^{-1}\right)\end{array}$} & \multicolumn{2}{|c|}{$\begin{array}{c}\text { Gain par rapport } \\
\text { à la « banane pérenne » } \\
\left(€ \cdot \mathrm{ha}^{-1} \cdot \mathrm{an}^{-1}\right)\end{array}$} \\
\hline & & Non subventionnée & Subventionnée \\
\hline Avec jachère non subventionnée & +450 & +900 & 0 \\
\hline Avec jachère subventionnée & +720 & +1170 & +270 \\
\hline
\end{tabular}

incluses dans les différents systèmes de culture, s'établissent à $1580 €$ pour une culture pérenne, $2030 €$ pour une culture de bananier en continu, $8960 €$ pour une culture en rotation et $3760 €$ pour une conduite de la bananeraie avec jachère ${ }^{7}$. Si le classement relatif de ces marges est robuste, leur valeur absolue doit être examinée avec précaution car elles sont essentiellement déterminées par les niveaux de rendements dont la précision résulte de l'évaluation de ces valeurs par les producteurs eux-mêmes. Cette analyse est cependant insuffisante car elle ne tient pas compte des coûts d'entretien de la jachère (respectivement des autres cultures composant le système cultural) et du manque à gagner sans production de banane (respectivement du gain des autres cultures du système cultural), ce qui implique un calcul pluriannuel. Pour combler ces lacunes, des calculs complémentaires ont été effectués à partir d'une analyse des actes techniques et de leur coût dans deux exploitations pratiquant la jachère.

Dans les systèmes bananiers replantés après jachère (mode de conduite "banane + jachère "), cette dernière a un coût total qui comprend un coût pour l'entretien (1470 $€ \cdot \mathrm{ha}^{-1}$ pour une jachère avec cyclonage des bananiers et arrachage des repousses et $1320 € \cdot h^{-1}$ pour une jachère avec piquage au glyphosate des bananiers) et un

\footnotetext{
${ }^{7}$ Nous ne tenons pas compte ici du fait que, pour un même système de culture, les marges dépendent des types d'exploitation [14]. dans le dispositif des CAD. L'aide à la mise en jachère est mobilisable dans le cadre du DOCUP depuis 2004.
}

manque à gagner dû au fait de l'absence de production de bananes pendant la durée de la jachère. En contrepartie, elle permet un gain de rendement, grâce à la restauration de la fertilité du sol, et des économies de charges (produits phytosanitaires) qui, en l'absence de subvention, se traduisent par une augmentation de la rentabilité par rapport aux modes de conduite "banane continue " et " banane pérenne " (tableau VI). Par ailleurs, il existe actuellement deux mesures, une aide à la bananeraie pérenne d'altitude (900 $€ \cdot \mathrm{ha}^{-1} \cdot \mathrm{an}^{-1}$ pendant 5 ans) et une aide à la mise en jachère avant replantation de bananeraie $^{8}$ (1890 € ha $^{-1}$ pour la durée de la jachère en 2004), par définition incompatibles l'une avec l'autre. Quels que soient les systèmes de culture et les combinaisons de subventions, l'avantage est toujours au système " bananier + jachère " (à l'exception de la bananeraie pérenne d'altitude subventionnée et de la bananeraie avec jachère non subventionnée qui sont équivalentes) (tableau VI). Cependant, la période de transition de plusieurs années, nécessaire pour passer du système pérenne au système avec jachère, est une étape à franchir : elle pose des problèmes de gestion de la main-d'œuuvre chez les bananiers de type "entrepreneurial " qui reposent sur de la main-d'œuvre salariée.

8 L'aide à la bananeraie pérenne d'altitude était mobilisable dans le cadre des CTE jusqu'en 2003, elle a ensuite été intégrée 
Elle a également un coût (manque à gagner et coût d'entretien d'une jachère), insurmontable pour les exploitants qui n'ont pas suffisamment de revenus et de disponibilité en trésorerie pendant la période de transition. Même si un calcul économique simple a montré que l'introduction d'une jachère permettait d'accroître la marge brute produite, seuls les producteurs qui ont les moyens de faire face à ces coûts pourront être concernés par ce changement de pratique. Par ailleurs, l'efficacité économique d'une jachère peut être remise en cause par une mauvaise maîtrise de la qualité de la jachère (maintien de plantes hôtes de nématodes) ou du matériel végétal (contamination des pépinières par des nématodes), susceptible de remettre en cause le gain en rendement de la bananeraie après jachère [33]. Ce risque intervient aussi dans le processus de prise de décision de l'exploitant, ce qui est un des facteurs pouvant expliquer le fait que la monoculture reste dominante dans les exploitations, malgré l'intérêt économique des pratiques de jachère.

\section{Discussion}

\subsection{Adéquation entre dispositifs d'appui et stratégies des agriculteurs}

Les objectifs sous-jacents aux référentiels techniques et aux subventions aux pratiques de jachère s'accordent avec ceux des exploitations centrées sur la production bananière disposant d'une forte technicité visant une augmentation du rendement. Cependant, un ensemble d'autres objectifs est laissé de côté : alimentation d'un troupeau, obtention d'un complément de revenu par la diversification, accompagnement de la reconversion de l'exploitation de la production bananière vers la diversification, etc. Il existe un décalage entre la fonction d'assainissement portée par un dispositif d'appui et les différentes fonctions attribuées par les agriculteurs à la jachère. Trois principales conséquences résultent de cette inadéquation entre les objectifs des dispositifs d'appui et ceux des agriculteurs :
- La première de ces conséquences concerne les dispositifs et leurs utilisations. Différents exemples montrent que les dispositifs d'appui ne sont pas utilisés tels qu'ils ont été conçus : demandes de dérogation en raison du non respect du cahier des charges pour obtenir l'aide " jachère " (demande de suppression de l'herbicide pour pâturage par les boufs), friches déclarées comme des jachères pour bénéficier des aides, vitroplants utilisés, car faciles à planter et subventionnés, mais non précédés d'une jachère ou d'une culture non hôte de nématodes, etc. Considérés comme détournement ou ajustement, ces exemples appellent une évolution des dispositifs d'appui.

- La seconde conséquence intéresse les mesures ou stratégies. Les objectifs qui ont conduit à soutenir financièrement la mesure " jachère raisonnée " touchent à la préservation de la fertilité des parcelles et visent implicitement la réduction de l'utilisation des produits phytosanitaires. Or, d'autres facteurs contribuent à la diminution de la charge phytosanitaire : à l'échelle de la parcelle, raisonnement des apports en fonction du niveau d'infestation parasitaire (par exemple à l'aide de pièges à charançon ou d'analyses nématologiques en culture bananière) ; au niveau d'un bassin versant, réglementation des apports en produits polluants afin de les éloigner des voies de transfert, sécurisation de l'acquisition du foncier afin de favoriser la mise en place de systèmes de culture raisonnés, etc. [34]. Les enquêtes réalisées montrent qu'indépendamment des effets de la jachère sur les populations de parasites, les agriculteurs s'engageant dans cette pratique réduisent d'eux-mêmes les doses en pesticides. La jachère est donc ici le reflet d'une stratégie plus globale visant la réduction des intrants. De ce point de vue, il peut paraître plus efficace de soutenir ces stratégies globales de protection de l'environnement plutôt qu'une mesure unique qui répondra plus difficilement aux attentes des agriculteurs.

- La troisième conséquence résultant de l'inadéquation entre les objectifs des dispositifs d'appui et ceux des agriculteurs implique de prendre en compte la diversité. L'adoption d'une pratique agricole plus respectueuse de l'environnement dépend du 
système de production [35]. La diversité des pratiques illustrée dans les résultats exposés appelle une diversification des référentiels techniques et des systèmes d'aide. Actuellement, les référentiels techniques disponibles ne couvrent qu'une partie des besoins des agriculteurs en s'adressant aux exploitations spécialisées dans la production de bananes. Des référentiels sont encore à élaborer (ou en cours d'élaboration), par exemple sur les effets assainissants des cultures fourragères ou sur les jachères permettant de gérer les incompatibilités entre cultures dans des rotations.

\subsection{Du transfert technique à l'accompagnement des producteurs}

L'identification des différents freins à l'évolution des pratiques de jachère permet d'évaluer les modifications qu'il est nécessaire d'apporter dans les dispositifs mis en œuvre pour l'accompagnement des producteurs. Ils ont trait au conseil, au système d'aide, à la gestion du risque et des aléas au sein des exploitations.

\subsubsection{Conseil et information de l'exploitant}

Actuellement, les supports techniques distribués aux planteurs se basent essentiellement sur des arguments agronomiques et commerciaux pour orienter les interventions sur les parcelles [11, 12, 36]. Or, l'étude fait ressortir le poids de l'environnement économique dans l'évolution d'assolements. Il apparaît en conséquence nécessaire de renforcer les incitations et les conseils, sans se limiter aux arguments agronomiques connus des exploitants, mais en les complétant par une démonstration des intérêts économiques et environnementaux au niveau de l'exploitation agricole.

\subsection{2. Évolution du système de crédit}

Même si globalement l'introduction d'une jachère apporte un supplément de revenu, elle induit également des charges ou manques à gagner auxquels certains exploitants ne peuvent pas faire face. Un système de crédit adapté paraît nécessaire pour faire face à ces difficultés. Nous avons vu précédemment (cf. Sect. 3.4.) l'importance de replacer les pratiques de jachère et rotation dans les stratégies productives des exploitants. Cela souligne la nécessité de passer d'un modèle centré sur le transfert de référentiels techniques à une approche construite autour de l'accompagnement des agriculteurs dans la réalisation de leurs projets. L'exemple que nous avons présenté (tableau VI) montre le poids potentiel des systèmes d'aide dans la prise de décision des exploitants. La production bananière guadeloupéenne est caractérisée par l'importance des transferts publics qui induisent des stratégies de captation d'aides. Une étude conduite dans les exploitations agricoles ayant signé des CTE "banane " l'illustre. Elle a montré que, dans ce cas, les exploitants cherchaient avant tout l'apport en trésorerie, sans projet d'exploitation construit [37]. La démarche d'accompagnement de projets d'agriculteurs n'est donc pas encore opérationnelle et manque d'outils, notamment de diagnostic d'exploitation.

\subsubsection{Gestion du risque et de l'aléa}

L'étude de la gestion du risque et de l'aléa fait ressortir le poids des aléas (économique, climatique) sur les stratégies d'exploitations. Ces aléas ne trouvent leur valorisation qu'à travers les systèmes d'indemnisation mis en place (indemnisation sécheresse en 2001 et 2002, cyclone, apurement des dettes, etc.). L'aléa est parfois intégré à la stratégie des exploitants : pour le choix des parcelles à mettre en jachère par exemple, un exploitant nous a déclaré : "Le cyclone décide pour moi. "Cependant, une meilleure valorisation de ces aléas pourrait être trouvée (par exemple en orientant les pratiques vers un assainissement des parcelles au lieu de laisser la bananeraie en friche à la suite de l'arrêt d'exploitation de cette culture) et soutenue par le conseil technique.

\section{Conclusion}

La caractérisation des pratiques de jachère et rotation nous a permis d'identifier des 
décalages entre préconisations et mise en œuvre. L'origine de ces décalages est à rechercher dans la diversité des pratiques mises en œuvre, qui reflète la variabilité des fonctions et objectifs attribués à la mise en place de ces systèmes de culture. Le contexte de forte pression sociétale pour une agriculture plus respectueuse de l'environnement conduit actuellement à soutenir des pratiques dont les effets bénéfiques sont avérés, plutôt que des stratégies globales de maîtrise des impacts environnementaux. Le risque réside dans la non-concordance des objectifs des dispositifs d'appui et de ceux des exploitants qui peuvent conduire à des dérives, voire des abus.

La caractérisation de la diversité des pratiques et l'identification des principaux freins à la mise en place de pratiques assainissantes permettent de proposer des adaptations ayant trait au conseil, au système d'aide, à la gestion du risque et des aléas au sein des exploitations. Ces analyses dépassent le simple cadre de la parcelle et de l'exploitation agricole et doivent considérer les interactions entre les différents usagers de l'espace, qui sont prises en compte dans les programmes de recherche en cours.

\section{Remerciements}

Nous remercions pour leur contribution à ce travail V. Amoravain, D. Duféal et J.C. Govindin pour la réalisation des diagnostics de territoire ; A. Berger et N. Girbal dans l'acquisition des références technico-économiques sur les jachères.

\section{Références}

[1] Sebillotte M., Jachère, système de culture, système de production, méthodologie d'étude, J. Agric. Tradit. Bot. Appl. 24 (1977) 241-264.

[2] Sebillotte M., Allain S., Dore T., Meynard J.M., La jachère et ses fonctions agronomiques, économiques et environnementales. Diagnostic actuel, Courr. Environ. Inra 20 (1993) 11-22.

[3] Jouve P., La jachère en Afrique de l'Ouest et au Maghreb, Jachères, Doss. environ. Inra 9, (1994) 161-170.
[4] Boserup E., Évolution agraire et pression démographique, Flammarion, Paris, France, 1970.

[5] Bellon S., Dore T., Chabert J.P., Barrio J., Agriculteurs et agronomes face à la politique agricole commune de jachère-gel, in: Proc. Colloq. Nouvelles fonctions de l'agriculture et de l'espace rural, Inra, Toulouse, France, 1996, pp. 139-158.

[6] Risède J.M., Tézenas du Montcel H., Systèmes monoculturaux bananiers et protection de l'environnement : état des lieux et perspectives, Fruits 52 (4) (1997) 225-232.

[7] Price N.S., Alternate cropping in the management of Radopholus similis and Cosmopolites sordidus, two important pests of banana and plantain, Int. J. Pest Manage. 40 (3) (1994) 237-244.

[8] Mateille T., Quénéhervé P., Hugon R., The development of plant-parasitic nematode infestations on micro-propagated banana plants following field measures in Côte d'Ivoire, Ann. Appl. Biol. 152 (1) (1994) 147159.

[9] Sarah J.L., Lassoudière A., Guérout R., La jachère nue et l'immersion du sol : deux méthodes intéressantes de lutte intégrée contre Rodopholus similis dans les bananeraies des sols tourbeux de Côte d'Ivoire, Fruits 38 (1) (1983) 35-41.

[10] Tian G., Kang B.T., Kolawole G.O., Idinoba P., Salako F.K., Long-term effects of fallow systems and lengths on crop production and soil fertility maintenance in West Africa, Nutr. Cycl. Agroecosys. 71 (2) (2005) 139-150.

[11] Anon., Restauration de la fertilité des sols en bananeraie : la pratique d'une jachère raisonnée, Fiche tech. $\mathrm{n}^{\circ} 1$, Cirad-Flhor, Neufchâteau, Guadeloupe, 2001, 6 p.

[12] Anon., Conduite au champ de vitroplants de bananiers, Fiche tech. $n^{\circ} 2$, Cirad-Flhor, Neufchâteau, Guadeloupe, 2001, 4 p.

[13] Papy F., Interdépendance des systèmes de culture dans l'exploitation, in: E. Malezieux, Trebuil G., Jaeger M., Modélisation des agroécosystèmes et aide à la décision, Cirad/Inra, Montpellier, France, 2001.

[14] Manceron S., MICA Zone bananière. Une représentation de la diversité des exploitations bananières. Des outils d'aide à la décision, Rapp. tech. final programme européen DOCUP 2000-2006 "Réalisation et diffusion du modèle de simulation MICA », volet « Zone bananière ", Inra, Guadeloupe, 2004, 56 p. 
[15] Anon., Recensement agricole 2000. L'inventaire. Département d'outre-mer, Ministère de l'Agriculture de l'alimentation de la pêche et de la ruralité, AGRESTE, Paris, France, 2000, CD Rom.

[16] Amoravain V., Analyse du système agraire sur un bassin-versant bananier en Guadeloupe, Cnearc-Isara-Cirad, Montpellier, France, 2000, 88 p.

[17] Duféal D., Diagnostic territorial en zone bananière. Étude d'une petite région rurale : la section de l'Habituée, Enita-Cirad, ClermontFerrand, France, 2001, 40 p.

[18] Govindin J.C., Diagnostic territorial en zone bananière, Univ. Antilles Guyane-Cirad, Pointe-à-Pitre, France, 2001, 89 p.

[19] Landais E., Typologies d'exploitations agricoles: nouvelles questions, nouvelles méthodes, Écon. Rural. (236) (1996) 3-27.

[20] Dulcire M., Cattan P., Monoculture d'exportation et développement agricole durable : cas de la banane en Guadeloupe, Cah. Agric. 11 (2002) 313-321.

[21] Mallessard R., Pre-diagnostic de la filière de production banane de Guadeloupe, CiradFlhor, Montpellier, France, 1998, 71 p.

[22] Dorel M., Enquête diagnostic en bananeraie. Étude des facteurs limitants, Cirad, Neufchâteau, Guadeloupe, 1988, 93 p.

[23] Melin P., Godefroy J., Dormoy M., Étude de la rotation culturale bananiers-aubergines dans les conditions écologiques du centre de la Martinique. Actions sur les caractéristiques chimiques, structurales et microbiologiques du sol, Fruits 43 (6) (1988) 349-352.

[24] Ternisien É., Melin P., Étude des rotations culturales en bananeraie. Première partie : bilan des cultures de rotation, Fruits 44 (7-8) (1989) 373-383.

[25] Ternisien É., Ganry J., Rotations culturales en culture bananière intensive, Fruits $\mathrm{n}^{\circ}$ spécial (1990) 98-102.

[26] Grugeaux-Etna M.F., Pollution de l'eau : quatre années de silence, Sept-Mag. 1089 (2000) pp. 11-13.
[27] Grugeaux-Etna M.F., Pollution des terres. Légumes empoisonnés sur les marchés, Sept-Mag. 1265 (2003) pp. 12-13.

[28] Balland P., Mestres R., Fagot M., Rapport sur l'évaluation des risques liés à l'utilisation de produits phytosanitaires en Guadeloupe et Martinique, Ministère de l'Aménagement du territoire et de l'environnement. Direction de l'Eau, Paris, France, 1998, 95 p.

[29] Bonan H., Prime J.L., Rapport sur la présence de pesticides dans les eaux de consommation humaine en Guadeloupe, Ministère de l'Aménagement du territoire et de l'environnement, Ministère de l'Emploi et de la solidarité, Paris, France, 2001, 77 p.

[30] Anon., Les pesticides dans les eaux. Cinquième bilan annuel. Données 2001, Inst. Fr. Environ., Orléans, France, 2003, 24 p.

[31] Chabrier C., Quénéhervé P., Control of the burrowing nematode (Radopholus similis) on banana: impact of the banana field destruction method on the efficiency of the following fallow, Crop Prot. 22 (1) (2003) 121-127.

[32] Torre A., Caron A., Conflits d'usage et de voisinages dans les espaces ruraux, Sci. Soc. 57 (2002) 95-113.

[33] Marie P., Culture raisonnée et réduction des coûts de production : vers une production bananière raisonnée et rentable, FruiTrop 74 (2000) 12-15.

[34] Houdart M., Filin Y., Saububray F., Réduction de la pollution des eaux par modification quantitative et spatiale de la charge polluante, Cah. PRAM 3 (2003) 20-23.

[35] Paineau F., Demazeau E., Bel M.P., Quels éléments conditionnent le regard des agriculteurs sur l'environnement et l'adoption des pratiques plus respectueuses de l'environnement ? Courr. Environ. Inra 35 (1998) 65-70.

[36] Anon., Le manuel du planteur, Sicabam, service agro-technique, Fort-de-France, Martinique, France, 1998.

[37] Gimenez G., Les CTE en Guadeloupe : impact sur les exploitations et conséquences pour le développement de l'agriculture, Enesad-Cirad, Mém., Neufchâteau, Guadeloupe, France, 2003, 59 p. 


\section{Convergencias y diferencias entre los objetivos de los dispositivos de apoyo y entre los de los agricultores: el caso de los barbechos en producción de plátanos en Guadalupe.}

Resumen - Introducción. En las Antillas, la introducción del barbecho, cuya utilidad se mostró en el control del parasitismo telúrico, está en el orden del día por el hecho de la explosión del parasitismo ligado a la monocultura platanera. Debido a que esta práctica está defendida por parte de la investigación y por parte de los poderes públicos, el estudio emprendido tuvo como finalidad el análisis de las diferencias entre preconizaciones manifestadas por el dispositivo institucional y entre las prácticas de los agricultores con el fin de definir en qué condiciones se pueden poner en pie las prácticas de barbecho. Metodología. Se abordaron a partir de sendas investigaciones junto a los cultivadores los determinantes y las funciones atribuidas a los barbechos, así como los frenos. Éstas permitieron recoger entre otros, variables necesarias para la caracterización de reglas de decisión en materia de gestión de las rotaciones de cultivos y de las obligaciones endógenas a la explotación. La elección de las explotaciones investigadas nos condujo a estudiar las prácticas de barbechos en el marco de una tipología de explotación distinguiendo, entre 42 explotaciones estudiadas, los tipos, "Diversificados", "Plátano familiar" y "Plátano empresaria". Estos tipos son representativos de las explotaciones de menos de 20 ha. El caso del barbecho en Guadalupe. Los resultados han mostrado que el barbecho está presente en más de la mitad de los cultivadores sondeados. Su situación varía con los tipos de explotación, bien está sometida, o bien está integrada en los sistemas de cultura. Los modos de comportamiento son funciones de los objetivos de los cultivadores: asaneamiento, pasto, gestión de la tesorería, etc. Los frenos hacia el desarrollo de los barbechos son numerosos: falta de superficie, dificultad de razonar a largo plazo, escasez de ganar en una tierra que no "produce", etc. No obstante, los cálculos económicos en sistemas de cultura que incluyen el barbecho muestran que sus márgenes brutos respectivos son superiores a aquellos encontrados para plantaciones bananeras continúas y perennes. La obligación principal se encuentra en el momento del cambio de sistema cultural cuando el cultivador debe confrontarse con una tierra que sólo aportará suplemento de rendimiento dos años más tarde. Discusión y conclusión. Nuestro estudio ha puesto en evidencia que la multiplicidad de las prácticas de barbecho, pertinentes en relación a los objetivos de los cultivadores, tan sólo está tomada en consideración parcialmente en los dispositivos institucionales existentes. Esto mismo exige una diversificación de las referencias técnicas.

Francia / Guadalupe / Musa / barbecho / cultivo / monocultivo 